Western University

Scholarship@Western

Aboriginal Policy Research Consortium International (APRCi)

7-13-2012

\title{
Growth and Empowerment for Indigenous Australians in Substance Abuse Treatment
}

Stacey L. Berry

T. P. Crowe

F.P. Deane

M. Billingham

Y. Bhagerutty

Follow this and additional works at: https://ir.lib.uwo.ca/aprci

Part of the Substance Abuse and Addiction Commons

Citation of this paper:

Berry, Stacey L.; Crowe, T. P.; Deane, F. P.; Billingham, M.; and Bhagerutty, Y., "Growth and Empowerment for Indigenous Australians in Substance Abuse Treatment" (2012). Aboriginal Policy Research Consortium International (APRCi). 245.

https://ir.lib.uwo.ca/aprci/245 


\title{
Growth and Empowerment for Indigenous Australians in Substance Abuse Treatment
}

\author{
Stacey L. Berry • T. P. Crowe • F. P. Deane • \\ M. Billingham • Y. Bhagerutty
}

(C) Springer Science+Business Media, LLC 2012

\begin{abstract}
This paper describes psychosocial outcomes of an Indigenous residential substance abuse rehabilitation centre in Australia, examines the sensitivity to change of the new Growth and Empowerment Measure (GEM), and explores the degree to which service users value cultural components of the treatment program. Participants were 57 Indigenous and 46 non-Indigenous male clients from Oolong House. Intake, 8-weeks, and 16-weeks (program completion) measures of Kessler 10 Psychological Distress Scale (K10), Drug Taking Confidence Questionnaire (DTCQ-8), and GEM were completed. The Treatment Component Evaluation (TCE) was completed at 16-weeks. There were significant improvements for participants, with a decrease in psychological distress and increases in refusal self-efficacy and empowerment. Effect sizes for GEM were medium to large across the time-points ( $r=0.61$ to 0.70 for all four subscales from baseline to 8-weeks; $r=0.44$ to 0.70 for three subscales from 8 -weeks to 16 -weeks), indicating sensitivity to change. Indigenous participants rated cultural components of treatment significantly more helpful than did non-Indigenous participants. Implications for future research and substance abuse interventions for Indigenous Australians are discussed.
\end{abstract}

Keywords Indigenous - Australian - Empowerment - Treatment effectiveness - Sensitivity to change $\cdot$ Culture

Since colonisation Indigenous Australians have experienced extreme levels of loss, grief, disempowerment, cultural alienation, and loss of identity (Hunter 1993). This has been the consequence of many years of systematic assault on their traditional practices, families, languages and cultures (Leenaars et al. 1999). Significant damage was caused during colonisation when governmental policies were implemented to displace Indigenous communities from their land and remove Indigenous children from their families (Dudgeon et al. 2010). These

S. L. Berry $(\bowtie) \cdot$ T. P. Crowe $\cdot$ F. P. Deane

Illawarra Institute for Mental Health, University of Wollongong, Building 22, Wollongong,

NSW 2522, Australia

e-mail: slb775@uowmail.edu.au

M. Billingham • Y. Bhagerutty

Oolong Aboriginal Corporation Incorporated, 11 Junction Street, Nowra, NSW 2541, Australia 
policies affected a disruption to the traditional way of life which has spanned more than two centuries so far, and the result has been a widespread severing of cultural and spiritual lines. Many Indigenous people's sense of identity, spiritual and physical wellbeing, and general psychological adjustment has been negatively impacted (The Human Rights and Equal Opportunity Commission 1997). This is evident through extremely poor physical health profiles, as well as prevalence rates higher than the Australian average for suicide, domestic violence, substance abuse, and unemployment (Cleworth et al. 2006). Please note that throughout this paper the term Indigenous Australians refers to both Aboriginal Australians and Torres Strait Islanders.

Although many Indigenous Australians refrain from drinking alcohol (Perkins et al. 1994), Indigenous people who do drink are more likely to do so at high-risk levels (Department of Health and Ageing 2007). The use of other substances such as opiates, cannabis, amphetamines, and injecting drugs is increasing in Indigenous populations (Australian Institute of Health and Welfare 2005, 2006; Brady 2002). Cultural alienation and loss of identity have consistently been stated to contribute to the relatively high prevalence of mental health disorders in Indigenous communities when compared with non-Indigenous communities (Australian Institute of Health and Welfare 2002, 2003; Human Rights and Equal Opportunity Commission 1997; O'Shane 1995; Parker 2010; Roxbee and Wallace 2003; Swan and Raphael 1995).

There is evidence that Indigenous Australians do not access mental health and substance abuse services at a level consistent with their level of need (Westerman 2004). Although many factors likely contribute to the relatively low levels of service utilisation, a major factor that has been proposed is the failure of health services to embrace an understanding of Indigenous conceptualisations of mental health (Berry and Crowe 2009), including the holistic nature of health and wellbeing (Ypinazar et al. 2007). The word punyu, from the language of the Ngaringman of the Northern Territory, explains that health encompasses both person and Country (Atkinson et al. 2002). Punyu is associated with being strong, happy, knowledgeable, beautiful, clean, socially responsible and safe (i.e. being within the law and also being cared for by others) (Mobbs 1991). The health of Indigenous Australians may not be considered in terms of a mind/body dichotomy, as it is generally viewed in a western model of health and illness (Slattery 1994). There are current attempts to define a therapeutic approach that accounts for the realities of Indigenous culture (Chenhall 2006).

Culture has been successfully incorporated into interventions for Native American populations (Edwards 2003; Gone 2011; Janelle et al. 2009; Kirmayer et al. 2003; Moran and Bussey 2007; Wendt and Gone 2012; Wright et al. 2011). The degree to which an individual is embedded in his/her cultural traditions (enculturation) is believed to serve an important protective function in relation to alcohol cessation and abstinence with Native American populations (Torres Stone et al. 2006). Enculturation is evidenced by use of traditional language, practices, spirituality, and cultural identity (Whitbeck et al. 2004; Zimmerman et al. 1994). Torres Stone and colleagues (2006) found that participation in traditional activities and traditional spirituality were associated with alcohol cessation. They also suggested that spirituality and participation in traditional practices may be better indicators of enculturation than cultural identity.

Many Indigenous Australians report a significant connection to their culture. Anderson (1996) suggests that for Indigenous people, "our identity as human beings remains tied to our land, to our cultural practices ... our intellectual traditions, our concepts of spirituality... Destroy this relationship and you damage — sometimes irrevocably_individual human beings and their health" (p. 15). Caring for Country, defined as having the knowledge and responsibility to manage traditional lands, has been found to foster self identity and build self esteem for Indigenous Australians (Kingsley et al. 2009). Also, reinvigoration of 
traditional lifestyle has been associated with significant health benefits for Indigenous Australians (Burgess et al. 2008; O'Dea 1984), and an association has been found between cultural attachment and positive outcomes in health, psychological wellbeing, substance abuse, and employment (Dockery 2009, 2011). It is important to identify what works in recovery from substance abuse for Indigenous Australians, including identifying cultural drivers of resilience and health gains (National Health and Medical Research Council 2002).

Empowerment-based approaches are recognised as important for assisting individuals and communities to become active agents in reducing health inequities (Laliberte et al. 2009). A reference group of Indigenous Australians described empowerment as "a healing journey through which individuals come to terms with their past, learn better ways to deal with their present situation, gain control, become strong, and find their voice to participate in change for a strong community" (Laliberte et al. 2009, p. 66). Laliberte and colleagues state that in the context of the Australian historical policies on forced displacement and relocation empowerment becomes an important tool to assist individuals and communities regain a sense of belonging and group identity. An intervention which aimed to enhance empowerment for members of the Yarrabah Men's Health Group found that the intervention led to significant changes in the men's personal development, growth, and response to family responsibilities (Tsey et al. 2003, 2004).

The Growth and Empowerment Measure (GEM: Haswell et al. 2010) is a tool developed by Indigenous Australians for Indigenous Australians. The GEM was developed to evaluate programs that aim to enhance empowerment, and it measures both the process and outcomes of empowerment. The GEM is relatively unique in its attempt to measure the process of empowerment (Haswell et al. 2010), as other measures of empowerment-like constructs have focused on measuring outcomes or indicators of change rather than processes (e.g. the sense of coherence scale; Antonovsky 1993). Contemporary views of recovery focus on aspects of well-being rather than exclusively on reduction in symptoms, and it has been argued that recovery from substance abuse must extend beyond the substance abuse behaviour to encompass a process of self-improvement and renewed life (Laudet 2007). In line with these views, the GEM seeks to measure individuals' own perspectives of their psychosocial wellbeing and empowerment at a personal, family, and organisational level. Unlike previous measures of empowerment, the GEM is intended to be used in a variety of settings rather than being tailored to a specific context (Haswell et al. 2010). The GEM has not previously been used within a substance abuse treatment setting, and no data on its sensitivity to change has previously been reported.

The current study aimed to, firstly, examine changes in psychosocial wellbeing among clients of an Indigenous residential substance abuse rehabilitation centre in Australia, and secondly, examine the sensitivity of the GEM for detecting psychosocial changes in Indigenous Australians. This research also explored the degree to which service users valued the cultural components of the treatment program relative to other treatment components. To date there has been limited research investigating what works in substance abuse treatment for Indigenous Australians. Furthermore, there has been no research measuring emotional empowerment associated with treatment, and cultural components of treatment have rarely been examined in relation to psychosocial outcomes for Indigenous clients.

It was expected that there would be a negative association between symptom distress and empowerment, and between symptom distress and relapse self-efficacy at each of the measurement time points, and that there would be statistically significant positive change on all measures for clients receiving the treatment. This was based on the expectation that there would be an overall improvement of clients' health and well-being during the treatment 
program. It was also expected that Indigenous clients would rate the cultural components of treatment as more helpful than would non-Indigenous clients.

\section{Methods}

Ethics

Ethics approval was obtained and procedures were followed in accordance with the standards of the Human Research Ethics Committee of the University of Wollongong.

\section{Setting and Treatment Program}

Oolong House is an Indigenous residential substance abuse treatment centre on the South Coast of New South Wales, Australia. It is a modified therapeutic community providing evidence-based treatments (e.g. cognitive behaviour therapy) and group-based interventions, including Alcoholics Anonymous (AA) and Narcotics Anonymous (NA). As well as these interventions, which provide the basis of the treatment program, Oolong House also uses a traditional holistic community-healing model, incorporating the Indigenous community in the healing process. The program involves participation in cultural activities as well as cultural education in the areas of ancestry, cultural respect, land and humanity, hunting and gathering, language, storytelling, cultural identity, traditional artwork, construction of traditional musical instruments and weapons, traditional music, cultural dance, and visiting culturally significant sites. Oolong House provides a 16-week treatment program for male clients of Indigenous and non-Indigenous backgrounds. All clients participate in all components (i.e. cultural and non-cultural) of the treatment program.

\section{Participants}

Participants were recruited via convenience sampling from clients engaged in residential substance abuse treatment at Oolong House. Participation was on a voluntary basis with informed consent provided. Participants included 57 Indigenous and 46 non-Indigenous males over 18 years of age. Data were collected at intake, 8-weeks, and 16-weeks (program completion). Attrition resulted in sample sizes of 50 ( 25 Indigenous, 25 non-Indigenous) and 34 (20 Indigenous, 14 non-Indigenous) at the time points 8-weeks and 16-weeks respectively. Attrition was due to clients choosing to leave the program (e.g. due to family responsibilities or unwillingness to adhere to the program structure) and eviction (e.g. for violations of the program rules, including drug use or violence). No clients refused to participate in the study. Follow up data for clients that did not complete the program was not available, and therefore results do not reflect the self-perceived health of the treatment non-completers.

\section{Measures}

\section{Psychological Distress-Kessler 10 Scale (K10)}

The K10 (Kessler et al. 2002) is a brief 10-item self-report questionnaire designed to measure the level of distress and severity of psychological symptoms. The K10 is used widely, including in the World Health Organization World Mental Health Survey, and 
commonly used as a clinical outcome measure (Brooks et al. 2006). The K10 has been found to have high internal consistency reliability (Cronbach's alpha $=0.93$ ), good precision in the 90th-99th percentile range of the population distribution (standard errors of standardized scores in the range $0.20-0.25$ ), as well as an ability to discriminate DSM-IV cases from non-cases (areas under the Receiver Operating Characteristic curve of 0.87 to 0.96) (Kessler et al. 2002). The yarning about mental health version of the K10 (Nagel and Thompson 2007), developed for use with Aboriginal Australians, was used in this research. This version contains the same wording as the original, with additional graphics to promote comprehension of the response scale. Participants respond on a 5-point Likert scale from none of the time to all of the time.

\section{Drug Taking Refusal Self-Efficacy_Drug Taking Confidence Questionnaire, Eight-Item Version (DTCQ-8)}

The DTCQ-8 (Sklar and Turner 1999) is an 8-item self-report measure adapted from the original 50-item questionnaire (DTCQ-50; Annis et al. 1997) which measures a person's self-efficacy in not drinking or taking drugs in specific high relapse-risk situations. Analyses have demonstrated that the DTCQ-50 has a stable factor structure and is a reliable measure of coping self-efficacy for use across a wide range of addictions (Annis et al. 1997; Sklar et al. 1998). The DCTQ-8 has been shown to correlate at 0.97 with the total DTCQ-50 score and to account for $95 \%$ of the variance in total DCTQ-50 scores (Sklar and Turner 1999). Therefore the DTCQ-8 has been assessed to be a reliable and valid indicator of drug taking refusal self-efficacy (Sklar and Turner 1999). Participants respond on a 6-point scale from 0 to 100 , where $0=$ Not confident at all and $100=$ Very confident.

\section{Empowerment-Growth and Empowerment Measure (GEM)}

Empowerment was measured using the GEM (Haswell et al. 2010). The GEM is a selfreport measure comprised of a 13-item Emotional Empowerment Scale (EES14) and 12 Empowerment Scenarios (12S). This tool was designed as a part of the Empowerment Research Program (a collaboration of the University of Queensland and James Cook University) to provide a measure of dimensions of empowerment that are important to Indigenous Australians, and was developed using in-depth interview data from 50 participants of the Family Wellbeing Empowerment Program who described their experience of empowering change (see Tsey et al. 2005). The instrument was examined and improved through workshops in Alice Springs, Yarrabah and Cairns, with the help of Indigenous consultants as well as researchers experienced in the measurement of complex psychological concepts (Haswell et al. 2010). This scale has been purposely designed to be visually attractive, interesting, and simple to complete. The GEM has been shown to have robust internal reliability for the EES14 $(\alpha=0.89)$ and the $12 \mathrm{~S}(\alpha=0.86)$, and the individual components and summary scores of this tool measure inter-related but distinct aspects of empowerment and wellbeing. The GEM comprises four subscales, two within each of the EES14 and the 12S. Firstly the EES14 comprises the "Inner Peace" subscale (IP: items 2, 3, 4, 10, 11, 12, 13, and 14) and the "Self-Capacity" subscale (SC: items 5, 6, 7, and 9). The $12 \mathrm{~S}$ comprises the "Healing and Enabling Growth" subscale (HG: scenarios 1, 2, 3, 5, 8, 10, and 11) and the "Connection and Purpose" subscale (CP: scenarios 4, 6, 7, 9, and 12). All items on the EES14 are rated on a 5-point scale falling between two extremes. For example, for item 13 the first point on the scale is I live in fear of what's ahead, while the last point on the scale is I feel safe and secure, I can face whatever is ahead. All scenarios on the $12 \mathrm{~S}$ are 
rated on a 7-point scale falling between two extremes. For example, for item 4 the first point on the scale is There are things I should change in my life to be healthier and happier, but it seems all too hard. I don't think I can change anything at this time, and the last point on the scale is I have gained skills and confidence and have succeeded in making many important changes in my life. I feel fully confident about my ability to make changes.

\section{Helpfulness of Treatment Components-Treatment Component Evaluation (TCE)}

This measure was developed for this study and aims to evaluate different aspects of the program from the clients' perspective. Participants complete the TCE at the completion of the 16 week program, and responses are rated on a 5-point Likert scale regarding the extent to which participants believe that different aspects of the treatment program were helpful $(1=$ unhelpful to $5=$ extremely helpful $)$. A total of 16 aspects of treatment are rated in the TCE, including the cultural components of the program, i.e. "how helpful did you find the cultural program (e.g. artwork, weaving, dance) in assisting you reach your recovery goals?"

\section{Statistical Analyses}

Due to a high attrition rate, independent samples t-tests were conducted to determine whether non-completion (i.e. leaving the program before the 8-week and 16-week time-points) was associated with baseline scores on the K10, the DTCQ-8, or the GEM. To investigate the association between symptom distress measures and empowerment and refusal self-efficacy measures at baseline, correlational analysis was conducted. Where assumptions for parametric analysis were violated a Kendall's tau-b correlation was used. Kendall's tau-b has been found to have several advantages over Spearman's $r$ when applied to data from psychiatric treatment settings (Arndt et al. 1999). Repeated measures analysis of variance (ANOVA) as well as a series of paired t-tests were conducted to analyse changes over the three time-points for the K10, DTCQ-8, and GEM. Where assumptions for parametric analyses were violated, nonparametric Friedman Two-Way ANOVA and Wilcoxon Signed Ranks tests were used respectively. Effect sizes were calculated to indicate the sensitivity to change of the GEM (Haswell et al. 2010). Effect sizes were calculated as recommended by Clark-Carter (2004) by converting $z$ into $r$ using the formula $r=$ the absolute value of $z \div \sqrt{(N-\text { Ties })}$. Effect size has been used as an indicator of sensitivity to change in previous studies regarding substance abuse treatment (e.g. Butler et al. 2006), and a high effect size is indicative of high sensitivity to change and utility of the tool. Cohen (1988) suggests that $r$ values greater than 0.5 may be considered large, greater than 0.3 may be considered medium, and greater than 0.1 may be considered small. Finally, exploratory analysis was conducted to examine client responses on the TCE. Independent samples t-tests were conducted to investigate whether there was a significant difference between Indigenous and non-Indigenous clients' ratings of the helpfulness of various components of treatment, particularly cultural components of treatment.

\section{Results}

Attrition

A high attrition rate was found, with 103 participants at baseline, 50 participants at 8-weeks, and 34 participants at 16-weeks. Independent samples t-tests were conducted to investigate whether there was a significant difference on the baseline scores for clients who left the 
program before the 8 -week and 16 week-time points when compared with clients who remained in the program. For the 8-week time point the assumptions of scale of measurement, independence, and homogeneity of variance were met. Results indicate that there was no significant difference for any of the baseline measures for clients who left the program before the 8 -week time point when compared with clients who remained in the program (all $p$ values $>$ 0.27 ). For the 16-week time point the assumptions of scale of measurement, independence, and homogeneity of variance were met. Results once again indicate no significant difference on any of the baseline measures for clients who left the program before the 16 week time point when compared with clients who remained in the program (all $p$ values $>0.27$ ), although the result approached significance for the Empowerment Scenarios (S12) of the GEM $(p=0.06)$. Clients who completed the program had higher baseline scores for the S12 than clients who left the program before 16-weeks, indicating that baseline empowerment levels may have been a factor in determining whether or not clients remained in the treatment program.

\section{Psychosocial Outcomes}

Correlational analysis was conducted to investigate the association between symptom distress measures, empowerment and refusal self-efficacy measures at baseline. The assumption of normality was violated, therefore a Kendall's tau-b correlation was used. Higher scores on the K10 indicate higher levels of psychological distress, whereas higher scores on the DTCQ-8 and the GEM indicate higher levels of refusal self-efficacy and empowerment respectively. Results are summarised in Table 1. As expected, a significant negative correlation was found between the K10 and the DTCQ-8, and between the K10 and all four subscales of the GEM. Significant positive correlations were found between the DTCQ-8 and all four subscales of the GEM. This indicates that more psychological distress is associated with less empowerment and less confidence to resist the urge to use drugs, and conversely that more empowerment is associated with more confidence to resist relapse.

A repeated measures ANOVA was conducted to analyse changes over time for the K10, the DTCQ-8, and the GEM subscales over three times points: baseline, 8-weeks and 16-weeks. Several variables did not meet the assumptions for parametric analysis, therefore the nonparametric Friedman Two-Way ANOVA was used. The assumptions of independence and scale of measurement were met. Results indicate a significant difference between scores at baseline, 8-weeks and 16-weeks on the K10 $\left(\chi_{F}^{2}=27.74\right.$ (corrected for ties), $d f=2, N$-ties $=$ $34, p=0.000)$, the DTCQ-8 $\left(\chi_{F}^{2}=36.02\right.$ (corrected for ties), $d f=2, N$-ties $=34, p=0.000$ ), and all four subscales of the GEM including IP $\left(\chi_{F}^{2}=26.34\right.$ (corrected for ties), $d f=2, N$-ties $=34$, $p=0.000), \mathrm{SC}\left(\chi_{F}^{2}=22.42\right.$ (corrected for ties), $d f=2, N$-ties $\left.=34, p=0.000\right), \mathrm{HG}\left(\chi_{F}^{2}=32.67\right.$

Table 1 Kendall's tau-b correlations between scores at baseline $(N=103)$ on outcome measures

\begin{tabular}{llllll}
\hline Measure & 2 & 3 & 4 & 5 & 6 \\
\hline 1. Psychological Distress (K10) & $-0.27^{* *}$ & $-0.52^{* *}$ & $-0.34^{* *}$ & $-0.41^{* *}$ & $-0.37^{* *}$ \\
2. Refusal self-efficacy (DTCQ-8) & - & $0.40^{* *}$ & $0.34^{* *}$ & $0.35^{* *}$ & $0.30^{* *}$ \\
3. Inner Peace (GEM) & - & - & $0.54 * *$ & $0.48^{* *}$ & $0.38^{* *}$ \\
4. Self-Capacity (GEM) & - & - & - & $0.43^{* *}$ & $0.40^{* *}$ \\
5. Healing \& Enabling Growth (GEM) & - & - & - & - & $0.49 * *$ \\
6. Connection and Purpose (GEM) & - & - & - & - & - \\
\hline
\end{tabular}

** Correlation is significant at the $p<0.01$ level 
(corrected for ties), $d f=2, N$-ties $=34, p=0.000)$, and $\mathrm{CP}\left(\chi_{F}^{2}=14.80\right.$ (corrected for ties), $d f=2$, $N$-ties $=34, p=0.001$ ). Follow-up pairwise comparisons using the Wilcoxon Signed Ranks test were conducted and results are summarised in Table 2 . There was a significant difference between baseline and 8-weeks on all measures. This indicates that from baseline to 8-weeks participants' psychological distress significantly decreased, while their confidence in resisting relapse and their empowerment significantly increased. For 8-weeks to 16-weeks, the assumptions of independence and scale of measurement were met and results are summarised in Table 3. There was a significant improvement in scores for the K10, DTCQ-8, and the GEM subscales IP and HG. This indicates that from 8-weeks to 16-weeks participants' psychological distress significantly decreased, while their confidence in resisting relapse significantly increased along with two aspects of their empowerment (i.e. Inner Peace and Healing and Enabling Growth). There was an increase for the subscale SC that approached significance and a non-significant increase for the subscale CP.

\section{Sensitivity to Change of K10, DTCQ-8, and GEM}

Effect sizes between baseline and 8-weeks were large for the K10 $(r=0.67)$, the DTCQ-8 ( $r=$ $0.73)$, and all four subscales of the GEM ( $\mathrm{IP}=0.62 ; \mathrm{SC}=0.70 ; \mathrm{HG}=0.69 ; \mathrm{CP}=0.61)$. Effect sizes between 8-weeks and 16-weeks were large for the subscales IP $(r=0.70)$ and $\mathrm{HG}(r=0.53)$, medium for the K10 $(r=0.41)$, the DTCQ-8 $(r=0.44)$ and the subscale SC $(r=0.44)$, and small for the subscale $\mathrm{CP}(r=0.20)$. The effect sizes for the GEM subscales indicate that the GEM was highly sensitive to change in the current substance abuse treatment population, particularly during the earlier half of the treatment program.

\section{Clients' Perspectives on the Value of Cultural Components}

Exploratory analysis was conducted to examine clients' responses on the TCE. Independent sample t-tests were conducted to investigate whether there was a significant difference

Table 2 Summary of results from Wilcoxon Signed Ranks tests comparing outcome scores at baseline and 8-weeks

\begin{tabular}{|c|c|c|c|c|c|c|c|c|c|}
\hline \multirow[t]{2}{*}{ Measure } & \multicolumn{2}{|c|}{ Baseline } & \multicolumn{2}{|c|}{ 8-weeks } & \multirow[t]{2}{*}{$N$-Ties } & \multirow[t]{2}{*}{ Mean Rank ${ }^{\mathrm{a}, \mathrm{b}}$} & \multirow[t]{2}{*}{$T$} & \multirow[t]{2}{*}{$z$} & \multirow[t]{2}{*}{$p$} \\
\hline & Mean & SD & Mean & SD & & & & & \\
\hline $\mathrm{K} 10$ & 25.60 & 9.41 & 17.08 & 7.12 & 48 & 26.05 & 134 & -4.66 & 0.000 \\
\hline DTCQ-8 & 62.20 & 30.63 & 85.74 & 21.54 & 41 & 21.92 & 72 & -4.65 & 0.000 \\
\hline IP & 3.48 & 0.92 & 4.29 & 0.75 & 46 & 24.29 & 158 & -4.18 & 0.000 \\
\hline $\mathrm{SC}$ & 3.93 & 0.79 & 4.53 & 0.66 & 41 & 22.09 & 88 & -4.46 & 0.000 \\
\hline $\mathrm{HG}$ & 4.14 & 1.18 & 5.30 & 1.01 & 47 & 25.97 & 115 & -4.75 & 0.000 \\
\hline $\mathrm{CP}$ & 4.74 & 1.26 & 5.47 & 1.03 & 43 & 23.72 & 139.5 & -4.03 & 0.000 \\
\hline
\end{tabular}

\footnotetext{
${ }^{\text {a }}$ Positive ranks where 8-weeks > baseline for DTCQ-8, IP, SC, HG, and CP

${ }^{\mathrm{b}}$ Negative ranks where baseline $>8$-weeks for K10

K10 Kessler 10 psychological distress scale

DTCQ-8 Drug Taking Confidence Questionnaire, eight-item version

$I P$ inner peace

$S C$ self-capacity

HG healing and enabling growth

$C P$ connection and purpose
} 
Table 3 Summary of results from Wilcoxon Signed Ranks tests comparing outcome scores at 8-weeks and 16-weeks

\begin{tabular}{|c|c|c|c|c|c|c|c|c|c|}
\hline \multirow[t]{2}{*}{ Measure } & \multicolumn{2}{|c|}{ 8-weeks } & \multicolumn{2}{|c|}{ 16-weeks } & \multirow[t]{2}{*}{$N$-Ties } & \multirow[t]{2}{*}{ Mean Rank ${ }^{\mathrm{a}, \mathrm{b}}$} & \multirow[t]{2}{*}{$T$} & \multirow[t]{2}{*}{$z$} & \multirow[t]{2}{*}{$p$} \\
\hline & Mean & SD & Mean & SD & & & & & \\
\hline $\mathrm{K} 10^{\mathrm{b}}$ & 17.08 & 7.12 & 14.71 & 4.84 & 30 & 17.10 & 123 & -2.26 & 0.02 \\
\hline DTCQ-8 ${ }^{\mathrm{c}}$ & 85.74 & 21.54 & 93.07 & 9.81 & 22 & 12.67 & 63 & -2.06 & 0.04 \\
\hline $\mathrm{IP}^{\mathrm{d}}$ & 4.29 & 0.75 & 4.65 & 0.41 & 24 & 13.53 & 29.50 & -3.45 & 0.001 \\
\hline $\mathrm{SC}^{\mathrm{e}}$ & 4.53 & 0.66 & 4.71 & 0.43 & 18 & 10.63 & 43.50 & -1.85 & 0.064 \\
\hline $\mathrm{HG}^{\mathrm{f}}$ & 5.30 & 1.01 & 5.69 & 0.85 & 32 & 19.23 & 105 & -2.98 & 0.003 \\
\hline $\mathrm{CP}^{\mathrm{g}}$ & 5.47 & 1.03 & 5.63 & 0.93 & 29 & 15.74 & 167.5 & -1.08 & 0.279 \\
\hline
\end{tabular}

${ }^{\text {a }}$ Positive ranks where 16-weeks $>8$-weeks for DTCQ-8, IP, SC, HG, and CP

${ }^{\mathrm{b}}$ Negative ranks where 8 -weeks $>16$-weeks for K10

K10 Kessler 10 psychological distress scale

DTCQ-8 Drug Taking Confidence Questionnaire, eight-item version

$I P$ inner peace

$S C$ self-capacity

$H G$ healing and enabling growth

$C P$ connection and purpose

between Indigenous and non-Indigenous clients' ratings of the helpfulness of cultural components of treatment. The assumption of normality was violated, and therefore the MannWhitney $U$ test was used. As expected, results indicate that Indigenous clients (Mean Rank= $19.78, n=20)$ rate the cultural components as significantly more helpful than non-Indigenous clients (Mean Rank=14.25, $n=14$ ) $U=94.50, z=-1.68$ (corrected for ties), $p=0.05$, one-tailed. On a 5 -point Likert scale ( $1=$ unhelpful, 5 = extremely helpful) Indigenous clients' mean rating for the helpfulness of cultural components was 4.25, while non-Indigenous clients' mean rating was 3.57.

Further exploratory analysis was conducted by calculating the mean of clients' TCE scores across all treatment components and investigating whether this varied significantly between Indigenous and non-Indigenous clients. Using the Mann-Whitney $U$ test no significant difference was found between Indigenous and non-Indigenous clients on their mean TCE score $(p=0.12)$. Further exploratory analysis was conducted using the Mann-Whitney $U$ test to investigate whether Indigenous and non-Indigenous clients differed in their response regarding the helpfulness of the other 15 components of treatment measured on the TCE. Item 15, "advice about money or employment", was the only other component of treatment on which Indigenous and non-Indigenous clients differed significantly in their ratings of helpfulness. This item was rated as significantly more helpful by Indigenous clients (Mean score $=3.70$, Mean Rank=20.50, $n=20$ ) than non-Indigenous clients (Mean score $=2.79$, Mean Rank=13.21, $n=14$ ) $U=80.00, z=-2.18$ (corrected for ties), $p=0.03$, two-tailed.

\section{Discussion}

This study examined psychosocial outcomes for Indigenous and non-Indigenous clients within a residential substance abuse treatment program. For clients who completed the 16-week 
treatment program there were statistically significant improvements, including a reduction in psychological distress, and enhancements in refusal self-efficacy and feelings of empowerment. Correlational analysis of baseline scores indicated that higher psychological distress was associated with lower refusal self-efficacy and lower empowerment, suggesting that as psychological distress decreases, refusal self-efficacy and empowerment may be expected to increase. As expected, there were improvements on all outcome measures over the course of treatment.

The research findings indicate that the GEM was highly sensitive to change in the current substance abuse population, supporting utility of the GEM as a tool for use in substance abuse treatment. Effect sizes for the GEM subscales were of similar magnitude to the K10 and the DTCQ-8, two widely used and well-established measures, indicating that the GEM's sensitivity to change is comparable with these tools. These findings contribute to previous psychometric research examining the validity of the GEM (Haswell et al. 2010), although the results are preliminary and should be interpreted cautiously.

Results indicate that the GEM subscales are effectively measuring inter-related but distinct aspects of empowerment. Effect sizes for the four subscales vary during the second half of the treatment program, and as such the results may suggest that the subscales are measuring different processes of empowerment with varying trajectories. Results indicate that Inner Peace continues to improve at an increasing rate throughout treatment, with a higher effect size from 8-weeks to 16-weeks than from baseline to 8-weeks. Healing and Enabling Growth, Self-Capacity, and Connection and Purpose also continue to increase throughout treatment, although the extent of this increase plateaus somewhat after the first half of treatment. Different effects between the first 8 weeks and the second 8 weeks of treatment may also be a function of variations in sample sizes.

The results indicate that Inner Peace may be an aspect of empowerment that can continue to grow relatively independent of other aspects of empowerment. The items included on the Inner Peace subscale include "dealing with anger", "feeling calm and relaxed", "feeling safe and secure", "feeling centred and focused", "confident", "happy with self and life", "feeling strong and full of energy", and "feeling skilful" (Haswell et al. 2010). The Inner Peace subscale captures more well-being versus ill health or symptom distress. It is possible that Inner Peace continued to grow within the current research population in part due to the physical circumstances of the participants (i.e. living within a residential treatment program). Residential treatment settings entail limited contact with everyday stressors, and provide a relatively safe and structured environment, scheduled activities to promote participation, as well as educational activities and skills enhancement (Brunette et al. 2001, 2004). Many of the items on the Inner Peace subscale may have been responded to positively by participants due to the physical and practical confines of their environment. The other subscales include items such as "satisfaction with opportunity" (subscale SC), "feeling valued" (SC), "having a voice" (HG), "improving relationships" (HG), "being able to change" (CP), and "respected in my workplace" (CP) (Haswell et al. 2010). It is possible that these items evidenced less change than the Inner Peace items (during the second 8 weeks of treatment) because participants had less opportunity to address these issues due to the restrictive nature of the treatment program and setting.

The effect size for Inner Peace from 8-weeks to 16-weeks was large, while the effect sizes for the K10 and the DTCQ- 8 were medium. This suggests that sensitivity to change on the Inner Peace subscale is good compared to other measures. It is also possible that the treatment program better targets the well-being outcomes than symptom distress outcomes. Either way, the results suggest that the Inner Peace subscale assesses outcome domains not captured by more traditional outcome measures. The GEM measures individuals' own 
perspectives of their psychosocial wellbeing and empowerment, and the Inner Peace subscale explained most of the variance in overall scores on the GEM in a previous study by Haswell and colleagues (2010). Measures of psychological distress (e.g. K10) tend to capture more transient or subjective experiences that may be more readily affected by external stressors or cues, whereas psychosocial well-being may be a more stable process. The effect sizes on the Inner Peace scale were maintained over time whereas they tended to decrease on the K10 and DTCQ-8. As noted, this may reflect different change trajectories in different outcome domains.

Indigenous clients indicated that they found the cultural components of treatment significantly more helpful than did non-Indigenous clients, although this difference was small in magnitude. There is now a need to look more systematically at the relationship between cultural activities and treatment outcomes. The culture as treatment hypothesis (Brady 1995) suggests that a return to traditional Indigenous cultural practices is sufficient for effecting recovery from substance abuse for many Indigenous individuals. There has been limited empirical research to support this hypothesis to date (Gone and Calf Looking 2011). More detailed data regarding participation in therapeutic cultural activities and clearer descriptions of the mechanisms of these cultural components in relation to specific treatment goals is needed. This will allow a better understanding of the types of cultural activities that are most beneficial for Indigenous clients.

It should also be noted that non-Indigenous clients reported the cultural components of treatment as helpful. This suggests that whether or not an individual client identifies as being Indigenous they may gain significant therapeutic benefits from engaging in cultural activities. Cultural activities, similarly to general non-cultural activities, are helpful in providing distraction from cravings (Beck et al. 1993). It is possible that cultural activities can provide further benefits over and above general non-cultural activities. In other samples receiving substance abuse treatment it has been found that the two things they most wanted to gain from treatment were "strengthening of self-esteem" and "tranquillity" (Schneider et al. 2004). Previous research has linked engagement in cultural activities with enhanced selfesteem and feeling "grounded" (Kingsley et al. 2009), which can be conceptually related to tranquillity. Cultural activities which are aimed at enhancing self-esteem and tranquillity are likely to benefit Indigenous and non-Indigenous clients alike. Future research may examine the factors which impact on the degree to which individual clients benefit from cultural components of treatment, and in doing so assist treatment programs to identify clients who will benefit most from involvement in cultural aspects of treatment. Examination of the effects of cultural activities should not be restricted to Indigenous clients. However, we may expect that the benefits of cultural engagement may be greater for Indigenous clients due to the historical context of displacement and disempowerment which exists within Australia. For example, Indigenous individuals engaging in caring for Country activities have expressed feeling a sense of belonging enhanced via an inherent sense of obligation to their Country, as well as a reconnection with their ancestors (Kingsley et al. 2009).

There are some notable limitations to this research. Firstly, outcomes were only measured for clients who remained in treatment. Although there we no major differences between completers and non-completers on baseline measures, it is likely that the results will be biased towards a more positive outcome picture for completers. Secondly, participants were recruited from only one substance abuse service, therefore limiting the generalisability of the findings. A related issue is the relatively small sample size, which when combined with the need to use nonparametric analyses, reduces the overall power of the study. Finally, the measurement of the helpfulness of culturally relevant treatment components was limited to a single item. There is a need to develop more detailed multi-item measures of treatment 
components that capture both cultural and other (non-cultural) aspects of treatment. The Aboriginal Cultural Engagement Survey (ACES; Berry et al. 2012) may be used in future research to more directly measure engagement in cultural activities.

Despite these limitations, as far as we are aware this is the first study of psychosocial outcomes in an Indigenous specific substance abuse treatment program in Australia. The aim was to establish the capacity to conduct such research and explore some of the associated measurement issues. An important finding was that the GEM appeared to be sensitive to change amongst those who remained in treatment. Further, preliminary data suggest there were indications that culturally relevant therapeutic activities were perceived as more helpful amongst those who identified themselves as Indigenous. This offers promise for future outcome research using these measures and which aims to better understand the role of culturally specific treatment components. The GEM shows promise as a measure that expands on empowerment constructs in Indigenous people, but there is a need to further assess its psychometric properties and utility in different contexts.

Acknowledgements (1) This research was supported by a grant from the New South Wales Health Department and the Network of Alcohol and other Drug Agencies-Non government organisation mental health and drug and alcohol research grants program 2007.

(2) The authors acknowledge the hard work and contributions of the staff at Oolong House, as well as those who developed the GEM and shared their knowledge with us so generously, in particular Melissa Haswell.

\section{References}

Anderson, P. (1996). Priorities in Aboriginal health. In G. Robinson (Ed.), Aboriginal health: Social and cultural transitions: proceedings of a conference, 29-31 September, 1995, The Northern Territory University, Darwin (pp. 15-18). Darwin: NTU Press.

Annis, H. M., Sklar, S. M., \& Turner, N. E. (1997). The drug-taking confidence questionnaire user's guide. Toronto: Addiction Research Foundation of Ontario.

Antonovsky, A. (1993). The structure and properties of the sense of coherence scale. Social Science and Medicine, 36, 725-733.

Arndt, S., Turvey, C., \& Andreason, N. C. (1999). Correlating and predicting psychiatric symptom ratings: Spearman's r versus Kendall's tau correlation. Journal of Psychiatric Research, 33, 97-104.

Atkinson, J., Graham, J., Pettit, G., \& Lewis, L. (2002). Broadening the focus of research into the health of Indigenous Australians. The Medical Journal of Australia, 177, 286-287.

Australian Institute of Health and Welfare. (2002). Australia's health 2002. Canberra: AIHW.

Australian Institute of Health and Welfare. (2003). Australia's health 2003. The sixth biennial welfare report of the Australian Institute of Health and Welfare. Canberra: AIHW.

Australian Institute of Health and Welfare (2005). 2004 National Drug Strategy household survey: detailed findings (Drug Statistics Series No. 16. Cat. No. PHE 66). Canberra: Australian Institute of Health and Welfare (AIHW).

Australian Institute of Health and Welfare (2006). Statistics on drug use in Australia (Drug Statistics Series No. 18. Cat. No. PHE 80). Canberra: AIHW.

Beck, A. T., Wright, F. D., Newman, C. F., \& Liese, B. S. (1993). Cognitive therapy of substance abuse. New York: The Guildford Press.

Berry, S. L., \& Crowe, T. P. (2009). A review of engagement of Indigenous Australians within mental health and substance abuse services. Australian e-Journal for the Advancement of Mental Health, 8, www.auseinet.com/ journal/vol8iss1/berry.pdf

Berry, S. L., Crowe, T. P., \& Deane, F. P. (2012). Preliminary development and content validity of a measure of Australian Aboriginal cultural engagement. Ethnicity and Health. doi:10.1080/13557858.2011.645157.

Brady, M. (1995). Culture in treatment, culture as treatment: a critical appraisal of developments in addictions programs for indigenous North Americans and Australians. Social Science and Medicine, 41, 1487-1498. 
Brady, M. (2002). Indigenous residential treatment programs for drug and alcohol problems: Current status and options for improvement. Discussion paper for Centre for Aboriginal Economic Policy Research, paper no. 236.

Brooks, R. T., Beard, J., \& Steel, Z. (2006). Factor structure and interpretation of the K10. Psychological Assessment, 18, 62-70.

Brunette, M. F., Drake, R. E., Woods, M., \& Hartnett, T. (2001). A comparison of long-term and short-term residential treatment programs for dual diagnosis patients. Psychiatric Services, 52, 526-528.

Brunette, M. F., Mueser, K. T., \& Drake, R. E. (2004). A review of research on residential programs for people with severe mental illness and co-occurring substance use disorders. Drug and Alcohol Review, 23, 471-481.

Burgess, C. P., Berry, H. L., Gunthorpe, W., \& Bailie, R. S. (2008). Development and preliminary validation of the 'Caring for Country' questionnaire: Measurement of an Indigenous Australian health determinant. International Journal for Equity in Health, 7(26), http://www.equityhealthj.com/content/7/1/26

Butler, S. F., Budman, S. H., McGee, M. D., Davis, M. S., Cornelli, R., \& Morey, L. C. (2006). Addiction severity assessment tool: development of a self-report measure for clients in substance abuse treatment. Drug and Alcohol Dependence, 80, 349-360.

Chenhall, R. (2006). Psychotherapy with indigenous Australians: group work in a residential alcohol and drug treatment setting. Psychotherapy in Australia, 13, 62-68.

Clark-Carter, D. (2004). Quantitative psychological research: A student's handbook (2nd ed.). New York: Psychology Press.

Cleworth, S., Smith, W., \& Sealey, R. (2006). Grief and courage in a river town: a pilot project in the Aboriginal Community of Kempsey, New South Wales. Australasian Psychiatry, 14, 390-394.

Cohen, J. (1988). Statistical power analysis for the behavioural sciences (2nd ed.). Hillsdale: Erlbaum.

Department of Health and Ageing (2007). Alcohol treatment guidelines for indigenous Australians. National Alcohol Strategy.

Dockery, M. (2009). Cultural wellbeing: The case of Indigenous Australians. Curtin Business School: Curtin University.

Dockery, M. (2011). Traditional culture and the wellbeing of Indigenous Australians: An analysis of the 2008 NATSSIS. Curtin Business School: Curtin University.

Dudgeon, P., Wright, M., Paradies, Y., Garvey, D., \& Walker, I. (2010). The social, cultural and historical context of Aboriginal and Torres Strait Islander Australians. In N. Purdie, P. Dudgeon, \& R. Walker (Eds.), Working together: Aboriginal and Torres Strait Islander mental health and wellbeing principles and practice (pp. 25-42). Australian Government Department of Health and Ageing.

Edwards, Y. (2003). Cultural connection and transformation: substance abuse treatment at Friendship House. Journal of Psychoactive Drugs, 35, 53-63.

Gone, J. P. (2011). The red road to wellness: cultural reclamation in a Native First Nations community treatment center. American Journal of Community Psychology, 47, 187-202.

Gone, J. P., \& Calf Looking, P. E. (2011). American Indian culture as substance abuse treatment: pursuing evidence for a local intervention. Journal of Psychoactive Drugs, 43, 291-296.

Haswell, M., Kavanagh, D., Tsey, K., Reilly, L., Cadet-James, Y., Laliberte, A., et al. (2010). Psychometric validation of the Growth and Empowerment Measure (GEM) applied with Indigenous Australians. Australian and New Zealand Journal of Psychiatry, 44, 791-799.

Human Rights and Equal Opportunity Commission. (1997). Bringing them home: A report of the National inquiry into separation of Aboriginal and Torres Strait Islander children from their families. Sydney: Human Rights and Equal Opportunity Commission.

Hunter, E. (1993). Aboriginal health and history. Power and prejudice in remote Australia. Melbourne: Oxford University Press.

Janelle, A., Laliberte, A., \& Ottawa, U. (2009). Promoting traditions: an evaluation of a wilderness activity among First-Nations of Canada. Australasian Psychiatry, 17, 108-111.

Kessler, R. C., Andrews, G., Colpe, L. J., Hiripi, E., Mroczek, D. K., Normand, S. L. T., et al. (2002). Short screening scales to monitor population prevalences and trends in non-specific psychological distress. Psychological Medicine, 32, 959-976.

Kingsley, J., Townsend, M., Phillips, R., \& Aldous, D. (2009). "If the land is healthy...it makes the people healthy": the relationship between caring for country and health for the Yorta Yorta Nation, Boonwurrung and Bangerang Tribes. Health \& Place, 15, 291-299.

Kirmayer, L., Simpson, C., \& Cargo, M. (2003). Healing traditions: culture, community and mental health promotion with Canadian Aboriginal peoples. Australasian Psychiatry, 11, S15-S23.

Laliberte, A., Haswell-Elkins, M., \& Reilly, L. (2009). The healing journey: empowering Aboriginal communities to close the health gap. Australasian Psychiatry, 17, S64-S67.

Laudet, A. B. (2007). What does recovery mean to you? Lessons from the recovery experience for research and practice. Journal of Substance Abuse Treatment, 33, 243-256. 
Leenaars, A. A., Anawak, J., Brown, C., Hill-Keddie, T., \& Taparti, L. (1999). Genocide and suicide among Indigenous people: the North meets the South. Canadian Journal of Native Studies, 9, 337-363.

National Health and Medical Research Council (2002). Report to the Aboriginal and Torres Strait Islander Research Agenda Working Group (RAWG): Final report of community consultations on the RAWG road map October 2002.

Mobbs, R. (1991). In sickness and health: The sociocultural context of Aboriginal wellbeing, illness and healing. In J. Reid \& P. Trompf (Eds.), The health of Aboriginal Australia (pp. 292-325). Sydney: Harcourt Brace Jovanovich.

Moran, J. R., \& Bussey, M. (2007). Results of an alcohol prevention program with Urban American Indian youth. Child and Adolescent Social Work Journal, 24, 1-21.

Nagel, T., \& Thompson, C. (2007). Yarning about mental health manual. Darwin: AIMHI, Menzies School of Health Research.

O’Dea, K. (1984). Marked improvement in carbohydrate and lipid metabolism in diabetic Australian Aborigines after temporary reversion to traditional lifestyle. Diabetes, 33, 596-603.

O'Shane, P. (1995). The psychological impact of white colonialism on Aboriginal people. Australasian Psychiatry, 3, 149-153.

Parker, R. (2010). Australian Aboriginal and Torres Strait Islander mental health: an overview. In N. Purdie, P. Dudgeon, \& R. Walker (Eds.), Working together: Aboriginal and Torres Strait Islander mental health and wellbeing principles and practice (pp. 3-11). Australian Government Department of Health and Ageing.

Perkins, J. J., Sansom-Fisher, R. J., Blunden, S., Lunnay, D., Redman, S., \& Hensely, M. J. (1994). The prevalence of drug use in urban Aboriginal communities. Addiction, 89, 1319-1331.

Roxbee, L., \& Wallace, C. (2003). Emotional and social well-being: national policy context. Australasian Psychiatry, 11, S45-S50.

Schneider, U., Kroemer-Olbrisch, T., Wedegartner, F., Cimander, K. F., \& Wetterling, T. (2004). Wishes and expectations of alcoholic patients concerning their therapy. Alcohol \& Alcoholism, 39, 141-145.

Sklar, S. M., \& Turner, N. E. (1999). A brief measure for the assessment of coping self efficacy among alcohol and other drug users. Addiction, 94, 723-729.

Sklar, S. M., Annis, H. M., \& Turner, N. E. (1998). Development and validation of the Drug Taking Confidence Questionnaire: a measure of coping self-efficacy. Addictive Behaviors, 22, 1-18.

Slattery, G. (1994). Transcultural therapy with Indigenous families: working with the belief system. Australian \& New Zealand Journal of Therapy, 8, 61-70.

Swan, P., \& Raphael, B. (1995). 'Ways forward': National consultancy report on Aboriginal and Torres Strait Islander mental health. Canberra: Australian Government Publishing Service.

Torres Stone, R. A., Whitbeck, L. B., Chen, X., Johnson, K., \& Olson, D. M. (2006). Traditional practices, traditional spirituality, and alcohol cessation among American Indians. Journal of Studies on Alcohol, 67, 236-244.

Tsey, K., Patterson, D., Whiteside, M., Baird, L., Baird, B., \& Tsey, K. (2003). Indigenous men taking their rightful place in society? A follow up report. Australian Journal for Rural Health, 11, 285-286.

Tsey, K., Wenitong, M., McCalman, J., Whiteside, M., Baird, L., Patterson, D., et al. (2004). A participatory action research process with a rural Indigenous men's group: monitoring and reinforcing change. Australian Journal of Primary Health, 10, 130-136.

Tsey, K., Whiteside, M., Daly, B., Deemal, A., Gibson, T., Cadet-James, Y., et al. (2005). Adapting the 'Family Well-being' empowerment program to the needs of remote indigenous school children. Australian and New Zealand Journal of Public Health, 29, 112-116.

Wendt, D. C., \& Gone, J. P. (2012). Rethinking cultural competence: insights from indigenous community treatment settings. Transcultural Psychiatry, 49, 206-222.

Westerman, T. (2004). Engagement of Indigenous clients in mental health services: what role do cultural differences play? Australian e-Journal for the Advancement of Mental Health, 3, 1-6.

Whitbeck, L. B., Chen, X., Hoyt, D. R., \& Adams, G. W. (2004). Discrimination, historical loss, and enculturation: culturally specific risk and resiliency factors for alcohol abuse among American Indians. Journal of Studies on Alcohol, 65, 409-418.

Wright, S., Nebelkopf, E., King, J., Maas, M., Ptel, C., \& Samuel, S. (2011). Holistic system of care: evidence of effectiveness. Substance Use and Misuse, 46, 1420-1430.

Ypinazar, V. A., Margolis, S. A., Haswell-Elkins, M., \& Tsey, K. (2007). Indigenous Australians' understandings regarding mental health and disorders. Australian and New Zealand Journal of Psychiatry, 41, 467-478.

Zimmerman, M., Ramirez-Valles, J., Washienko, K. M., Walter, B., \& Dyer, D. (1994). The enculturation hypothesis: Exploring direct and positive effects among Native American youth. In H. I. McCubbin, E. A. Thompson, A. I. Thompson, \& J. E. Fromer (Eds.), Resiliency in ethnic minority families: Native and immigrant American families, vol. 1 (pp. 199-200). Madison: Centre for Excellence in Family Studies, University of Wisconsin-Madison. 